Vancomycin versus cefazolin prophylaxis for cardiac surgery in the setting of a high prevalence of methicillinresistant staphylococcal infections. $J$ Thorac Cardiovasc Surg 2002;123:326-332.

\section{Postoperative Bacteremia Secondary to Surgical-Site Infection}

Petti and colleagues from Duke University Medical Center, Durham, North Carolina, evaluated all surgical-site infections and postoperative bacteremias secondary to surgical-site infections as part of an ongoing active surgical surveillance program at a community hospital. Among 40,191 surgical procedures, they identified 515 patients with surgical-site infection and 47 with postoperative bacteremia secondary to surgical-site infection. Four variables were examined as potential predictors of the development of postoperative bacteremia secondary to a surgical-site infection: National Nosocomial Infections Surveillance System risk index, abdominal surgery, surgical procedures with an implantable device, and the presence of Staphylococcus aureus in wounds.

Of these four variables, only $S$. aureus isolated from a wound culture was associated with an increased risk for development of postoperative bacteremia secondary to surgical-site infection. Patients with $S$. aureus isolated in either pure or mixed culture from surgical-site infections were more than twice as likely as those without $S$. aureus wound infection to have postoperative bacteremia secondary to surgical-site infection.

FROM: Petti CA, Sanders LL, Trivette SL, Briggs J, Sexton DJ. Postoperative bacteremia secondary to surgical site infection. Clin Infect Dis 2002;34:305-308.

\section{Improving the Sensitivity of Direct Microscopy for the Detection of Acid- Fast Bacilli in Sputum}

Farnia and colleagues from Tehran, Iran, conducted a study to try to improve the results of direct smear microscopy. They used the mucus-digesting quality of chitin in tuberculosis (TB) laboratories. For this purpose, a total of 430 sputum specimens were processed by the Nacetyl-L-cysteine concentration, sodium hypochlorite $(\mathrm{NaOCl})$ liquefaction, chitin sedimentation, and direct microscopy methods. Then, the smear sensitivity for acidfast bacillus detection by chitin-treated sputum was compared with the sensitivities of smears prepared by other methods. The results showed that the chitin solution took less time to completely homogenize the mucoid sputum than did the $\mathrm{N}$-acetyl-L-cysteine and $\mathrm{NaOCl}$ methods. The $\mathrm{N}$-acetyl-L-cysteine concentration method demonstrated sensitivity and specificity levels of $83 \%$ and $97 \%$, respective- ly. In comparison, the sensitivity of chitin sedimentation was $80 \%$, with a specificity of $96.7 \%$. The $\mathrm{NaOCl}$ liquefaction method showed a sensitivity of $78 \%$, with a specificity of $96 \%$.

Finally, the sensitivity of direct microscopy was lower than those of the other tested methods, being only $46 \%$ with a specificity of $90 \%$. The chitin and $\mathrm{NaOCl}$ liquefaction methods are both easy to perform, and they do not require additional equipment (centrifuges).

The results also demonstrated that the chitin method is less time-consuming than the $\mathrm{NaOCl}$ method. Only 30 minutes of incubation is required to bring complete sedimentation of bacilli in chitin-treated sputum, whereas the $\mathrm{NaOCl}$ method requires 10 to 12 hours to give the same results in the same sputum specimens. Therefore, the chitin liquefaction and sedimentation method may provide better results in TB laboratories of developing countries than the N-acetyl- $\mathrm{L}$ cysteine concentration, $\mathrm{NaOCl}$ overnight sedimentation, and direct smear microscopy methods.

FROM: Farnia P, Mohammadi F, Zarifi Z, et al. Improving sensitivity of direct microscopy for detection of acid-fast bacilli in sputum: use of chitin in mucus digestion. $J$ Clin Microbiol 2002;40:508-511.

\section{Environmental Mycobacteria Can Cause Mycobacterium bovis BCG Vaccine to Fail}

The efficacy of Mycobacterium bovis bacillus CalmetteGuerin (BCG) vaccine against pulmonary tuberculosis (TB) varies enormously in different populations. The prevailing hypothesis attributes this variation to interactions between the vaccine and mycobacteria common in the environment, but the precise mechanism has not been clarified. Brandt and colleagues from the Statens Serum Institut, Copenhagen, Denmark, conducted a study that demonstrates that prior exposure to live environmental mycobacteria can result in a broad immune response that is recalled rapidly after BCG vaccination and controls the multiplication of the vaccine. In these sensitized mice, BCG elicits only a transient immune response with a low frequency of mycobacterium-specific cells and no protective immunity against TB. In contrast, the efficacy of TB subunit vaccines was unaffected by prior exposure to environmental mycobacteria. Six different isolates from soil and sputum samples from the Karonga district in Northern Malawi (a region in which BCG vaccination has no effect against pulmonary TB) were investigated in the mouse model, and two strains of the $M$. avium complex were found to block BCG activity completely.

FROM: Brandt L, Feino Cunha J, Weinreich Olsen A, et al. Failure of the Mycobacterium bovis BCG vaccine: some species of environmental mycobacteria block multiplication of BCG and induction of protective immunity to tuberculosis. Infect Immun 2002;70:672-678. 